\title{
Simultaneous cardiac and lung perforation by bone cement after percutaneous kyphoplasty
}

soon jin kim ${ }^{1}$ and kyung hwa kim²

${ }^{1}$ Jeonbuk National University Medical School, Jeonbuk National University Hospital

${ }^{2}$ Jeonbuk National University Medical School, Jeonbuk National University Hospital.

September 17, 2020

\begin{abstract}
Intracardiac cement embolism(ICE)-related cardiorespiratory complications may be delayed, and in the presence of symptomatic migrating bone cement, surgical retrieval must be considered to prevent progression to constrictive pericarditis and to relieve symptoms. This is the first report describing simultaneous cardiac and lung perforation caused by bone cement located in the right atrium after percutaneous vertebroplasty.
\end{abstract}

\section{Hosted file}

jcs manuscript.doc available at https://authorea.com/users/359697/articles/481528simultaneous-cardiac-and-lung-perforation-by-bone-cement-after-percutaneous-kyphoplasty
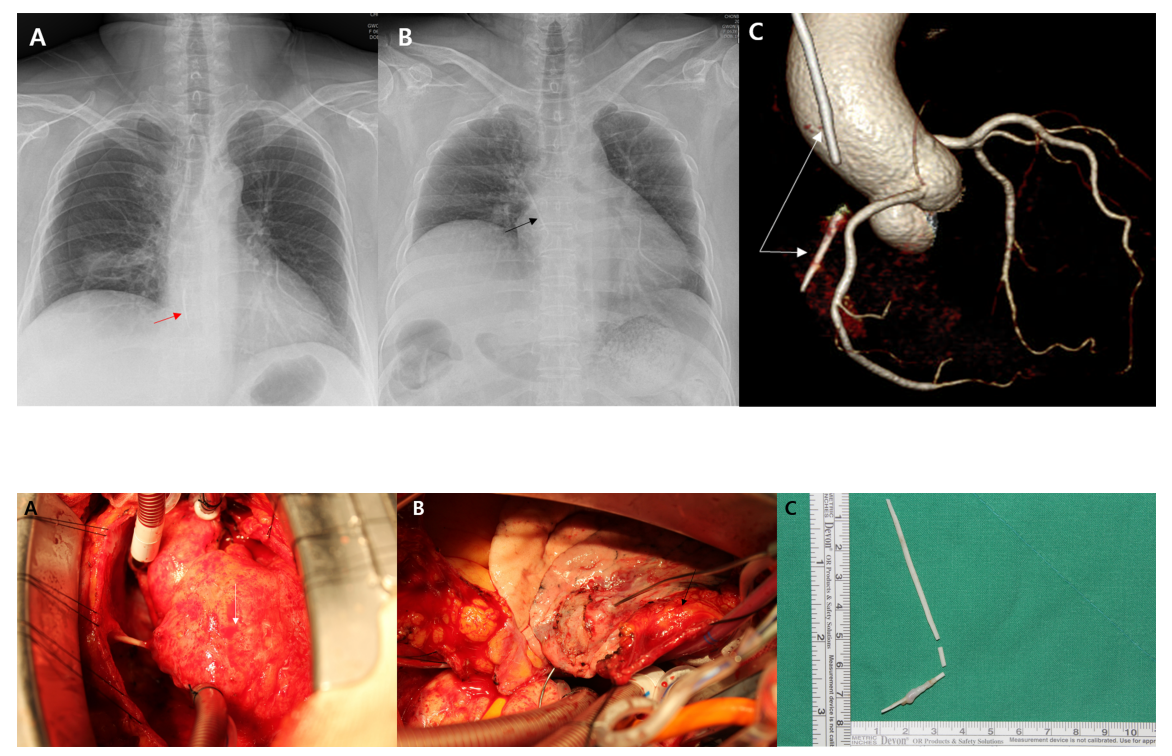